\title{
Intravascular Hemolysis in Aortic Stenosis
}

\author{
Akihiro TsuJI, Masaki TANABE, Katsuya OnISHI*, Tetsuya KitAMURA, \\ Tsutomu OKINAKA, Masaaki ITO, Naoki IsAKA and Takeshi NAKANO
}

\begin{abstract}
A 67-year-old woman with rheumatic aortic stenosis for 20 years was admitted to our hospital. Although she had no overt symptoms, she had severe aortic valve stenosis with a transvalvular pressure gradient of more than $150 \mathrm{mmHg}$. She had also been suffering from anemia and mild chronic renal failure. A peripheral blood smear showed numerous fragmented erythrocytes. Hemoglobin was $8.4 \mathrm{~g} / \mathrm{dl}$, lactate dehydrogenase was 316 IU/l, haptoglobin was less than $7.3 \mathrm{mg} / \mathrm{dl}$, and hemosiderinuria was evident. We diagnosed intravascular hemolysis related to aortic stenosis. After we performed an aortic valve replacement, fragmentation on the peripheral blood smear dramatically disappeared.

(Internal Medicine 43: 935-938, 2004)
\end{abstract}

Key words: intravascular hemolysis, aortic stenosis, native valve

\section{Introduction}

Intravascular hemolysis is caused by intracardiac flow disturbances in which high-velocity jets increase shear forces and disrupt erythrocyte membranes. The most common cardiovascular cause of intravascular hemolysis is paravalvular insufficiency around a prosthetic valve (1). However, there are few reports of intravascular hemolysis related to insufficiency or stenosis of the native valve (2).

\section{Case Report}

A 67-year-old woman with rheumatic aortic stenosis for 20 years and a history of infective endocarditis of the aortic valve 16 years previously was admitted to our hospital because of progression of her aortic stenosis. She had also been suffering from anemia and mild chronic renal failure due to nephrosclerosis. She had no history of overt congestive heart failure, syncope, or angina. On admission, her blood pressure was $150 / 60 \mathrm{mmHg}$, and her pulse rate was regular at 90 beats per minute. Her carotid upstrokes were reduced in volume and delayed. Her lungs were clear to auscultation. A 4/6 ejection systolic murmur could be heard at the right sternal border of the second intercostals space. The chest radiograph showed cardiomegaly without pulmonary congestion, and the electrocardiogram showed normal sinus rhythm with left axial deviation and strain pattern (Fig. 1). Two-dimensional echocardiography demonstrated increased aortic valve thickness and mild calcification in 3 cusps and commissural fusion as well as reduced leaflet excursion with systole. There were no signs of valve perforation or vegetation on the aortic valve. The left ventricular wall was thickened and wall motion was normal, and the mitral valve was almost intact. The transvalvular peak pressure gradient estimated by Doppler echocardiography was $160 \mathrm{mmHg}$, and the aortic valve area estimated on the basis of the continuity equation was 0.82 $\mathrm{cm}^{2}$ with mild aortic regurgitation (Fig. 2A). Coronary angiography revealed no significant stenotic lesions. Left ventricular systolic pressure was $310 \mathrm{mmHg}$, and the "peak to peak" pressure gradient obtained from the pull back method through the aortic valve demonstrated a pressure gradient of $150 \mathrm{mmHg}$ (Fig. 2B). Hemoglobin was $8.4 \mathrm{~g} / \mathrm{dl}$, mean corpuscular volume was $92.7 \mathrm{fl}$, mean corpuscular hemoglobin was $29.1 \mathrm{pg}$, and reticulocyte count was $22 \%$. Creatinine was $2.9 \mathrm{mg} / \mathrm{dl}$, and lactate dehydrogenase was $316 \mathrm{IU} / l$ (normal, 100-230), serum iron was $60 \mu \mathrm{g} / \mathrm{dl}$, and ferritin was $29.4 \mathrm{ng} / \mathrm{dl}$. A peripheral blood smear displayed numerous fragmented erythrocytes, haptoglobin was less than $7.3 \mathrm{mg} / \mathrm{dl}$, and hemosiderinuria was seen, suggesting intravascular hemolysis (Fig. 3A). The direct and indirect Coombs tests were negative, coagulation profiles were within normal limits, and ultrasonography showed that the spleen size was also in the normal range. Her anemia was diagnosed as a combination of iron deficiency, renal, and intravascular hemolytic anemia.

Although she had no overt symptoms related to aortic

From the First Department of Internal Medicine and *Department of Laboratory Medicine, Mie University School of Medicine, Mie

Received for publication November 28, 2003; Accepted for publication May 31, 2004

Reprint requests should be addressed to Dr. Masaki Tanabe, the First Department of Internal Medicine, Mie University School of Medicine, 2-174 Edobashi, Tsu 514-8507 


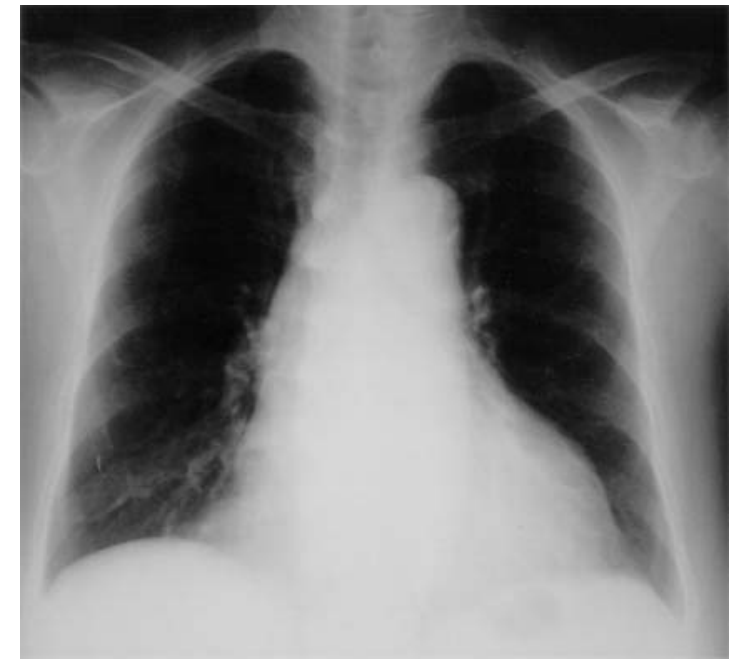

A

Figure 1. Chest radiograph and electrocardiogram on admission. A. Chest radiograph shows cardiomegaly without pulmonary congestion. B. Electrocardiogram shows left axial deviation and left ventricular hypertrophy.
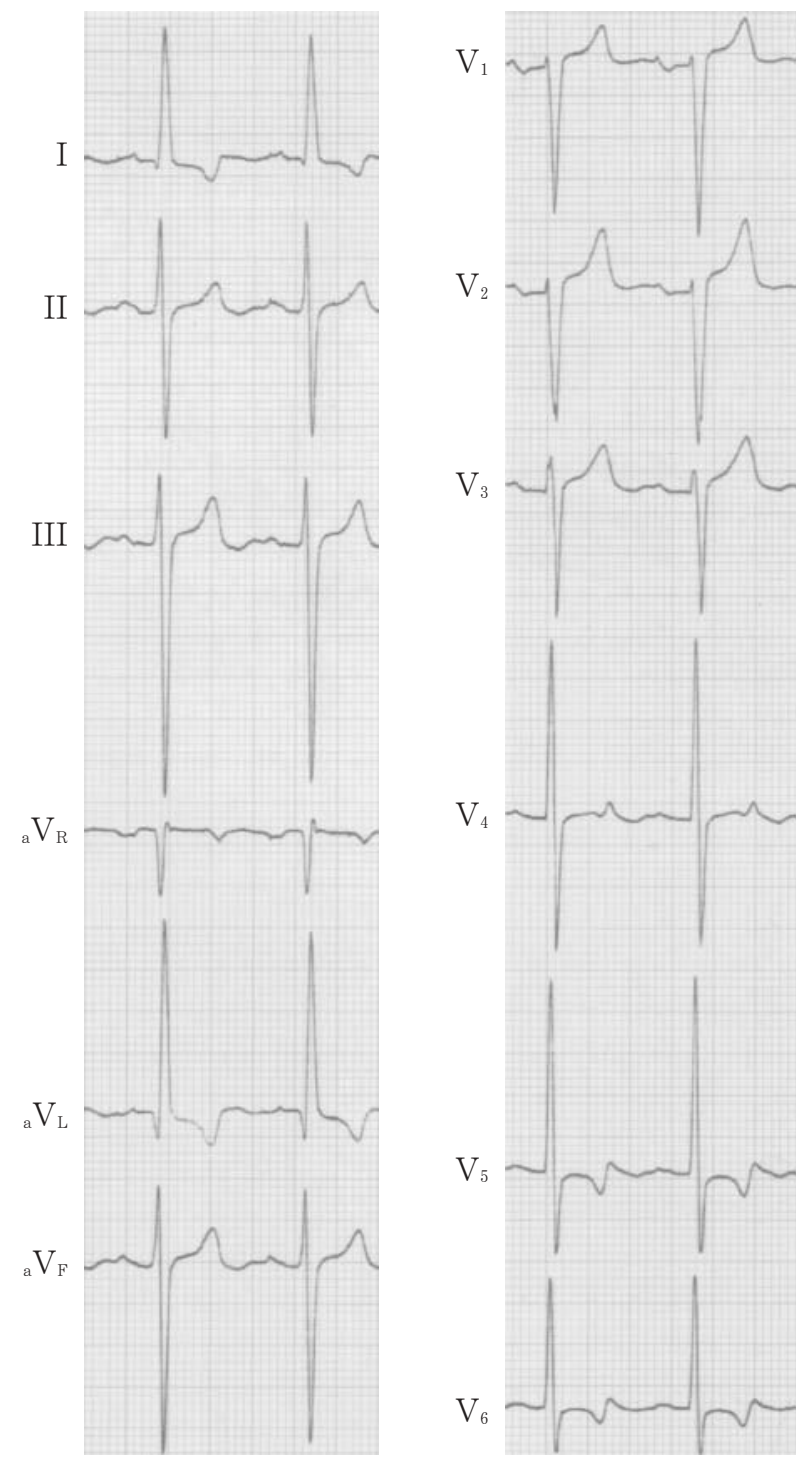

B

\section{Discussion}

Intravascular hemolysis, which is one cause of anemia, occurs often in patients with mechanical heart valve prostheses. However, there are few reports of intravascular hemolysis in patients with a disease in the native valve. Although significant hemolysis related to native valves is rare, cardiologists should consider the possibility of intravascular hemolytic anemia in patients with valvular heart disease.

Intravascular hemolysis is caused by intracardiac flow disturbances. In such disturbances high-velocity jets increase shear forces that disrupt erythrocyte membranes and result in hemolysis. Intravascular hemolysis may occur with hemolysis-prone erythrocytes or, if shear forces are great enough, with normal erythrocytes (1). 


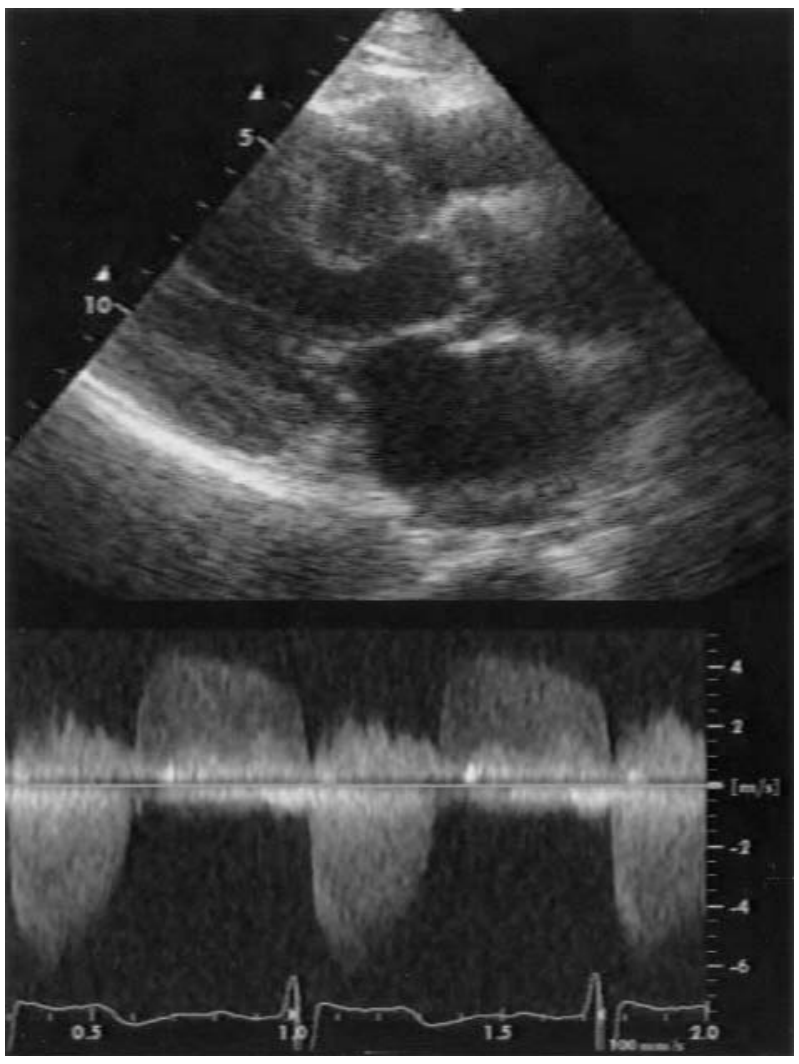

A

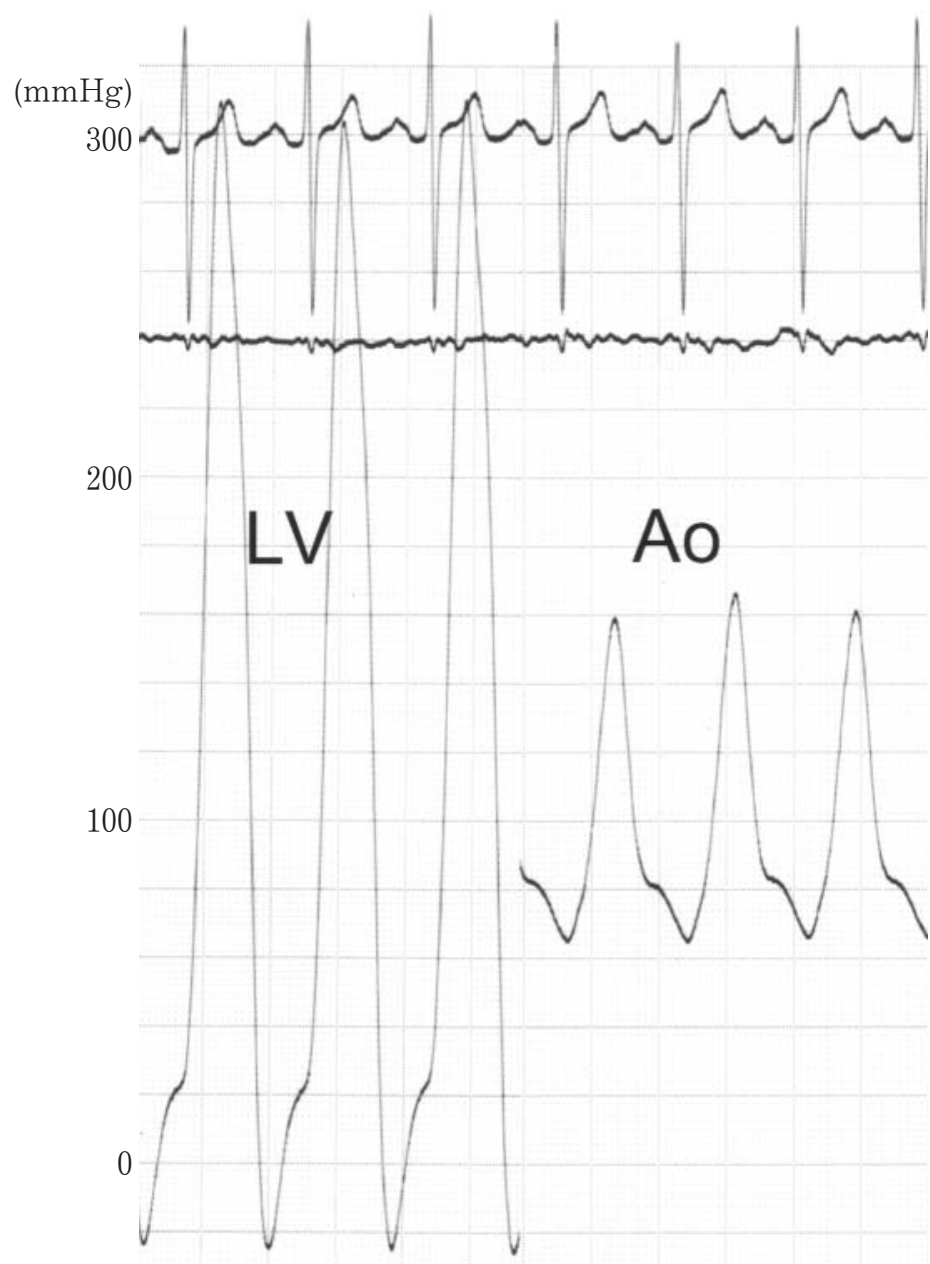

B

Figure 2. Echocardiogram, and left ventricular and aortic pressure tracings. A. Parasternal long-axis view shows left ventricular hypertrophy and a thickened aortic valve in the upper panel. Continuous-wave Doppler signal of the aortic valve from the apex shows aortic stenosis with $6.3 \mathrm{~m} / \mathrm{s}$ peak velocity $(160 \mathrm{mmHg}$ pressure gradient) and aortic regurgitation in the lower panel. B. Left ventricular (LV) and aortic pressure (Ao) obtained by the pull back method through the aortic valve demonstrated a pressure gradient of $150 \mathrm{mmHg}$.

Jacobson et al (2) reported that intravascular hemolysis develops when pressure gradients of $50 \mathrm{mmHg}$ or more occurred in aortic stenosis. When a pressure gradient of more than $50 \mathrm{mmHg}$ is present across a valve, shearing stress can exceed 4,000 dynes $/ \mathrm{cm}^{2}$. Theoretically, a shearing stress of more than 3,000 dynes $/ \mathrm{cm}^{2}$ can cause fragmentation of red blood cells (2-4). The present patient's pressure gradient was more than $150 \mathrm{mmHg}$, which was potentially high enough to cause hemolysis.

In addition to the importance of the pressure gradient to hemolysis, we must consider the effect of our patient's history of infective endocarditis. There are reports of fragmentation hemolysis related to acute infective endocarditis of the native valve $(4,5)$. The present case was not comparable with previous reports because the patient had had infective endocarditis 16 years previously. This had been resolved with antibiotics, and there was no valvular vegetation or perforation of the aortic valve at surgery or upon histologic examination. Changes at the valvular surface due to infection might enhance the shearing stress across the valve and contribute to hemolysis, although the aortic valve showed thickening and stiffening with little calcification and there were no specific findings related to hemolysis. Therefore, we speculated that rather than changes in the valvular structure, the severe pressure gradient itself may be one of the main causes of hemolysis in the present case.

Intravascular hemolysis is diagnosed by the presence of abnormal erythrocyte morphology on the blood smear, increased lactate dehydrogenase, reduced serum haptoglobin, and urinary hemosiderin. If the rate of hemolysis exceeds the 


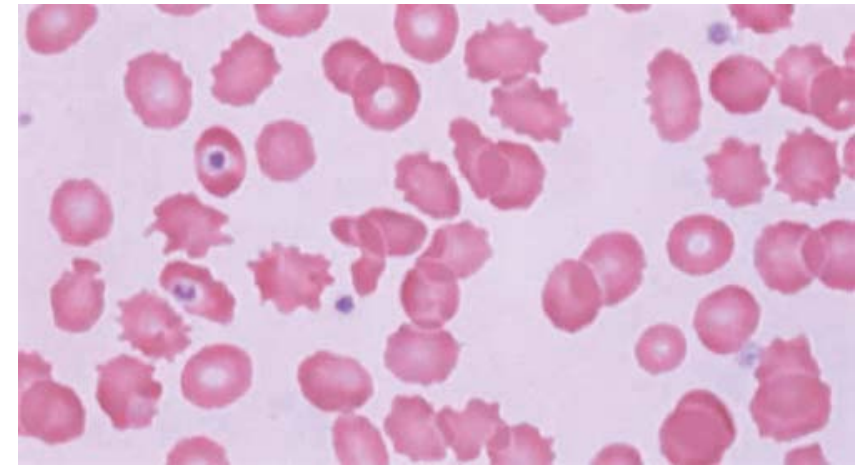

A

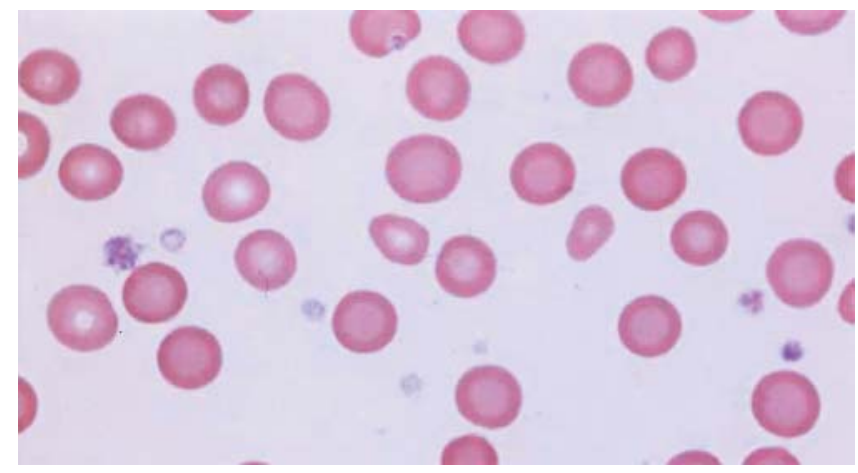

B

Figure 3. Peripheral blood smear stained with May-Giemsa. A. There were numerous fragmented erythrocytes in the peripheral smear before aortic valve replacement. B. Fragmented erythrocytes disappeared after aortic valve replacement. bone marrow reserve, anemia develops $(1,6)$. We diagnosed intravascular hemolysis related to aortic stenosis because our case had all of the above findings along with a severely stenotic aortic valve. However, confirming the main cause of anemia was difficult because several causes of anemia were present. The patient had renal insufficiency and iron deficiency, both of which can contribute to anemia. Although the contribution of hemolysis to anemia was unclear, confirming the presence of hemolysis was important to understanding the patient's condition and planning a therapeutic strategy.

The present case suggests the importance of considering valve hemolysis even in a patient with a native valve, when the patient has both valvular heart disease and anemia.

\section{References}

1) Hutchison SJ, Chandraratna PA. Hematologic diseases. in: Cardiology. Crawford MH, DiMarco JP, Asplund K, et al, Eds. Mosby, New York, 2001: 8.8.1-8.8.8.

2) Jacobson RJ, Rath CE, Perloff JK. Intravascular haemolysis and thrombocytopenia in left ventricular outflow obstruction. Br Heart $\mathrm{J}$ 35: 849-854, 1973.

3) Nevaril CG, Lynch EC, Alfrey CP Jr, Hellums JD. Erythrocyte damage and destruction induced by shearing stress. J Lab Clin Med 71: 784790, 1968.

4) Gradon JD, Hirshbein M, Milligan J. Fragmentation hemolysis: An unusual indication for valve replacement in native valve infective endocarditis. South Med J 89: 818-820, 1996.

5) Huang HL, Lin FC, Hung KC, Wang PN, Wu D. Hemolytic anemia in native valve infective endocarditis: A case report and literature review. Jpn Circ J 63: 400-403, 1999.

6) Udden MM. Hemolytic anemia: intravascular. in: Cecil Textbook of Medicine. 21st ed. Goldman L, Bennett JC, Eds. Saunders, Philadelphia, 1999: 882-884. 